\title{
Rosuvastatin exerts favourable effects on thrombosis and neointimal growth in a mouse model of endothelial injury
}

\author{
Katrin Schäfer, Kilian Kaiser, Stavros Konstantinides \\ Department of Cardiology and Pulmonary Medicine, Georg August University of Goettingen, Goettingen, Germany
}

\begin{abstract}
Summary
Apart from reducing systemic lipid levels, statins may improve the clinical course of atherosclerosis by exerting favourable pleiotropic effects on the vessel wall.We studied the effects of rosuvastatin, a new, potent 3-hydroxy-3-methylglutaryl coenzyme A (HMG-CoA) reductase inhibitor, on vascular remodelling after endothelial injury in the hyperlipidaemic apolipoprotein E-knockout (apoE-/-) mouse. ApoE-/- mice, 22-weeks-old, were injected daily with rosuvastatin at a low (I $\mathrm{mg} / \mathrm{kg} ; \mathrm{n}=27)$ or high dosage $(10 \mathrm{mg} / \mathrm{kg} ; \mathrm{n}=24)$, or with vehicle alone $(n=26)$.After treatment for 2 weeks, endothelial injury and thrombosis of the carotid artery was induced with 10\% ferric chloride. Treatment was then resumed for a 3-week period.AIthough statin treatment did not affect the plasma lipid levels of mice, mean times to arterial thrombosis were prolonged in the low-dose and the high-dose group compared to controls
\end{abstract}

\section{Keywords}

Animal models, atherosclerosis, tissue remodelling, fibrinogen / fibrin, restenosis

Thromb Haemost 2005; 93: $145-52$
$(P<0.05$ and $P<0.01$ respectively). Interestingly, rosuvastatin withdrawal 4 days before injury completely reversed the antithrombotic effects of the drug. In follow-up studies 3 weeks after injury, deposition of fibrin in the vessel wall was significantly reduced in the rosuvastatin-treated animals. There was an increase in the content of $\alpha$-actin-positive smooth muscle cells $(P=0.008)$ and collagen fibers $(P<0.00 \mathrm{I})$, and a concomitant decrease in the number of oxLDL-containing macrophages $(P<0.00 \mathrm{I})$. Overall, the neointimal area and the severity of luminal stenosis were significantly reduced in statin-treated mice. Thus, rosuvastatin attenuates arterial thrombosis and neointima formation, and it may stabilise vascular lesions developing after endothelial injury in mice. These effects are independent of systemic lipid lowering.

\section{Introduction}

Large-scale clinical trials have consistently demonstrated the efficacy of 3-hydroxy-3-methylglutaryl coenzyme A (HMG-CoA) reductase inhibitors (statins) in the primary and secondary prevention of cardiovascular disease due to atherosclerosis and its complications (1). According to the prevailing hypothesis, which has provided the basis for current clinical recommendations (2), the reduction in the rate of cardiovascular events in patients treated with statins is largely attributed to the potent lipid-lowering effects of these drugs. Interestingly, data from in vitro studies suggest that statins may also exert pleiotropic favourable effects on endothelial cells, smooth muscle cells and monocytes (3), and that they may modulate inflammatory and immune responses (4). However, the implications of these findings for human dis- ease remain uncertain (5), despite the accumulating evidence that the clinical benefits of statin treatment are not confined to patients with elevated cholesterol levels $(6,7)$.

Apolipoprotein E-knockout (apoE-/-) mice are characterised by markedly elevated plasma cholesterol and triglyceride levels which are not reduced by short-term (8) or long-term (9) statin treatment. Therefore, the apoE-/- mouse may be a powerful tool for dissecting the (possible) pleiotropic effects of statins on vascular homeostasis and, particularly, on atherogenesis and the wound healing process after injury. In one study, treatment of apoE-/- mice with simvastatin reduced aortic atherosclerosis (quantified through the measurement of aortic cholesterol content) apparently without affecting lesion histology (9), while another study found an increase of innominate/brachiocephalic artery atherosclerosis, but reported a more "favourable" lesion his- 
tology with reduction in intraplaque haemorrhage and calcification (10). Finally, a third study reported a reduction of atherosclerosis (aortic plaque area) in LDL receptor-knockout mice as opposed to an increase in plaque size in their apoE-knockout counterparts placed on high-dose simvastatin treatment (11). Technical difficulties in standardisation of mouse atherosclerosis as well as the different statin dosage and localisation of the vessels studied were probably responsible, at least in part, for these seemingly inconsistent findings, as they were, for example, in previous studies which attempted to clarify the role of fibrinolytic factors in atherosclerosis (12-14).

Atherothrombosis is not only a complication of plaque rupture, but also an important pathomechanism of atherosclerosis progression (5). In fact, thrombosis and thrombus organisation appear to be key events in vascular wall inflammation and the response to endothelial injury (15-17). Although experimental models of arterial injury and thrombosis cannot exactly reproduce the pathophysiology of human atherosclerosis, we and others have previously shown that the ferric chloride model of carotid artery injury results in a reproducible thrombotic reaction and the subsequent formation of neointimal lesions which exhibit several histological characteristics of human atherosclerotic plaques $(18,19)$. Therefore, in the present study, we employed the ferric chloride model in apoE-/- mice in order to study the effects of rosuvastatin, a new, potent HMG-CoA reductase inhibitor (20), on the vascular remodelling process. Our hypothesis was that rosuvastatin directly (i.e., independent of its lipidlowering properties) modulates thrombosis and the ensuing inflammatory response, and that these effects might contribute to a reduction in the size and an increase in the stability of neointimal lesions developing in mice after endothelial injury.

\section{Materials and methods}

\section{Rosuvastatin treatment and arterial injury in vivo}

Male and female apolipoprotein E-knockout (apoE-/-) mice were purchased from Jackson Laboratories (Bar Harbor, ME, USA). After weaning at the age of 3-4 weeks, mice were fed regular rodent chow. At the age of 18 weeks, mice were divided into three treatment groups. The first (control) group ( $\mathrm{n}=26 ; 12$ male, 14 female) received $100 \mathrm{~mL}$ normal saline (vehicle), the second group ( $\mathrm{n}=27 ; 14$ male, 13 female) was treated with rosuvastatin at a low dosage $(1 \mathrm{mg} / \mathrm{kg}$ body weight (BW) in $100 \mathrm{~mL}$ normal saline), and the third ( $\mathrm{n}=24 ; 14$ male, 10 female) receiv- ed a high dosage of rosuvastatin $(10 \mathrm{mg} / \mathrm{kg} \mathrm{BW})$. Rosuvastatin or vehicle was given to mice once daily by intraperitoneal injection. The investigators performing the in vivo experiments, and the platelet aggregation and immunohistochemical studies (described below) were blinded to the type of treatment.

After two weeks of treatment, mice were anaesthetised by intraperitoneal injection of xylazine/ketamine and subjected to carotid artery injury using ferric chloride according to a standardised protocol $(18,21)$. Briefly, the left carotid artery of the anaesthetised mouse was carefully exposed by blunt dissection, and a $0.5 \times 1.0-\mathrm{mm}$ strip of Whatman no. 1 filter paper soaked in $10 \%$ $\mathrm{FeCl}_{3}$ solution was applied to the surface of the vessel for $3 \mathrm{~min}$ utes. Carotid blood flow was monitored before and over a 30-minute period after the injury using an ultrasound flow probe (0.5 VB, Transonic Systems) interfaced with a flowmeter (T106, Transonic Systems) and a data acquisition program (WinDaq Lite, DATAQ Instruments). Complete thrombotic occlusion was considered to occur when flow decreased to $0.0 \pm 0.2 \mathrm{ml} / \mathrm{min}$ according to the manufacturer's specifications. After injury, treatment was continued over a 3-week period, i.e. throughout the vascular healing process (15).

In order to study the effects of acute statin withdrawal on arterial thrombosis after endothelial injury in vivo, an additional group of 18-week-old apoE-/- mice $(\mathrm{n}=12)$ received rosuvastatin at a high dosage $(10 \mathrm{mg} / \mathrm{kg})$ for 2 weeks. Subsequently, rosuvastatin treatment was withdrawn and mice were subjected to carotid artery injury with ferric chloride 4 days later under exactly the same experimental conditions as described above.

All animal care and experimental procedures were approved by the Animal Research Committee of the University of Goettingen and complied with national guidelines and the Guide for the Care and Use of Laboratory Animals published by the National Institutes of Health (NIH Publication No. 85-23, revised 1996).

\section{Body weight and metabolic plasma parameters}

Mice were weighed daily from the beginning of rosuvastatin (or vehicle) treatment at $18 \mathrm{WOA}$ until completion of the study at the age of 23 weeks. At the time of sacrifice, blood was collected from deeply anaesthetised mice by cardiac puncture in 3.8\% sodium citrate (final dilution). Plasma was obtained by centrifugation at 3,000 rpm for 5 minutes. Plasma total cholesterol, LDL and HDL cholesterol, and triglyceride levels were determined enzymatically (Sigma) (18).

\begin{tabular}{lccc|}
\hline & Control Group & $\begin{array}{c}\text { Low-Dose } \\
\text { Rosuvastatin }\end{array}$ & $\begin{array}{c}\text { High-Dose } \\
\text { Rosuvastatin }\end{array}$ \\
\hline Body weight $(\mathrm{g})$ & $26.2 \pm 5.1$ & $28.1 \pm 6.0$ & $26.9 \pm 5.6$ \\
Plasma triglycerides $(\mathrm{mg} / \mathrm{d} \mathrm{l})$ & $111 \pm 13$ & $105 \pm 3$ & $112 \pm 7$ \\
Plasma total cholesterol $(\mathrm{mg} / \mathrm{dl})$ & $220 \pm 62$ & $273 \pm 69$ & $282 \pm 67$ \\
Plasma LDL cholesterol $(\mathrm{mg} / \mathrm{dl})$ & $128 \pm 21$ & $104 \pm 18$ & $102 \pm 27$ \\
Plasma HDL cholesterol $(\mathrm{mg} / \mathrm{dl})$ & $44 \pm 2$ & $37 \pm 5$ & $39 \pm 10$ \\
\hline The differences between the groups did not reach statistical significance. Plasma parameters were \\
determined in 10 mice per group.
\end{tabular}

Table I: Body weight and metabolic plasma parameters (mean+S.D.) of apoE-/mice aged 23 weeks at the time of tissue harvest (after 5 weeks of statin treatment). 
Morphometric and histochemical analysis of neointima For tissue harvest and processing, anaesthetised animals were carefully perfusion-fixed with $4 \%$ zinc formalin through the left ventricle. The carotid artery including the bifurcation was excised, post-fixed in 4\% zinc formalin, dehydrated and embedded in paraffin wax.

For morphometric analysis, paraffin-embedded carotid artery sections were stained with Verhoeff's elastic stain and analysed (ImagePro Plus; Media Cybernetics). Five sections equally spaced throughout the injured segment (at 200- $\mu \mathrm{m}$ intervals) were evaluated and the results were averaged for each animal $(15,18,22)$. Groups of 18 to 21 animals were compared.

Interstitial collagen was detected by picrosirius red polarisation microscopy $(22,23)$. Immunohistochemistry was performed on $5 \mu \mathrm{m}$-thick paraffin sections as described $(15,22)$. Smooth muscle cells (SMC) were detected using a monoclonal anti-mouse $\alpha$-actin antibody (HRP-labeled; Dako) followed by incubation with aminoethyl-carbazole (AEC) reagent (Zytomed). Macrophages were identified using a rat anti-mouse Mac-3 antibody (PharMingen), and fibrinogen was localised with a rabbit anti-human fibrinogen antibody (Dako). Finally, oxidised LDL was detected using an antibody against murine malondialdehyde (MDA)-conjugated LDL (kind gift of Dr. W. Palinski, University of California, San Diego, CA, USA) and an avidin-biotin phosphatase detection system (Universal APAAP kit; Dako).

To quantitatively assess the presence of macrophages in the vessel wall, the area of the arterial wall (within the external elastic lamina) staining positive for Mac-3 antigen was expressed as percentage of the total area within the external elastic lamina. Similarly, the relative area of the arterial wall positive for $\alpha$-actin, oxLDL, fibrinogen and collagen was determined by dividing the positively stained area by the total area using the computer morphometry system (22) .

\section{In vitro platelet aggregation}

Preparation of platelet-rich plasma (PRP) and platelet aggregation studies were performed as previously described (24). For each experiment, blood was pooled from five to six animals that had been treated with low-dose or high-dose rosuvastatin, or with vehicle, for 14 days. A standard platelet concentration of $5 \times 10^{7} / \mathrm{ml}$ was used, and aggregation experiments were performed on a microplate reader. Briefly, a 5-mL volume of ADP solution at various concentrations $(0.01 \mu \mathrm{M}$ to $10 \mu \mathrm{M})$ was placed in each well, followed by the addition of $95 \mu \mathrm{L}$ of freshly prepared PRP. Changes in light transmission over time were expressed as percentage of platelet-poor plasma (PPP) light transmission, with transmission of untreated PRP serving as the zero standard. All experiments were performed in triplicate. Standard software programs were used to construct aggregation traces.

\section{Statistical analysis}

Comparisons between means of treatment groups were performed using the Kruskall-Wallis non-parametric test followed by Dunn's multiple comparison test. For comparisons of proportions, Fisher's exact test was used. All statistical tests were twosided with a $P$ value of $<0.05$ indicating statistical significance.

\section{Results}

\section{Effects of statin treatment and statin withdrawal on ar- terial thrombosis in vivo}

In agreement with previous reports $(9,10)$, treatment with rosuvastatin over a 5 -week period (from the $18^{\text {th }}$ to the $23^{\text {rd }} \mathrm{WOA}$ ) did not significantly alter body weight or plasma cholesterol or triglyceride levels in the apoE-/- mice (Table 1). After induction of carotid artery injury, thrombotic occlusion failed to occur in 4 of 26 mice (15\%) in the control group compared to 8 of 27 mice $(30 \%)$ in the low-dose rosuvastatin group ( $P=0.36$ vs. controls) and 9 of 24 mice $(38 \%)$ in the high-dose group $(P=0.15)$. The mean time to thrombotic occlusion was dose-dependently prolonged under rosuvastatin treatment, increasing from $14.2 \pm 1.1$ minutes (median, 11.6) in the control group to $17.5 \pm 1.0$ minutes (median, 15.5) in the low-dose $(P<0.05)$ and $18.4 \pm 1.2$ minutes (median, 16.2) in the high-dose group $(P<0.01$; Fig. 1A). At the end of the 30-minute flow monitoring period after injury, vascular patency (including both the vessels that never occluded and those that recanalised after thrombotic occlusion) was $39 \%$

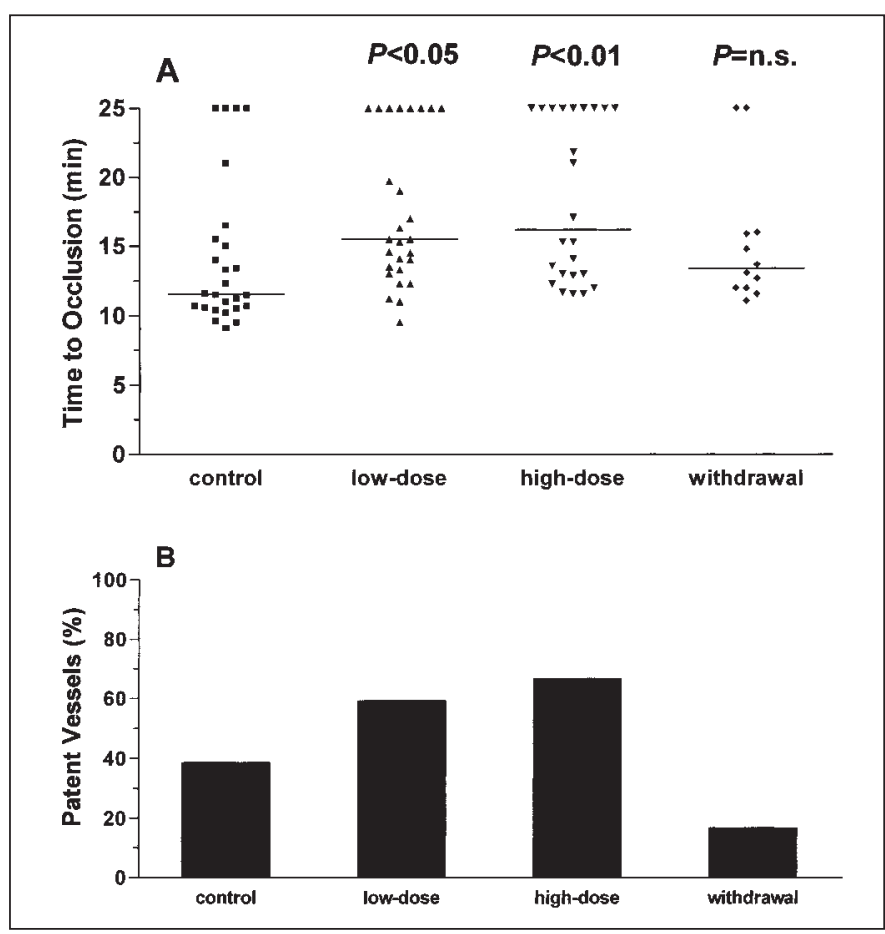

Figure I: Rosuvastatin prolongs the time to thrombotic occlusion (A) and increases arterial patency rates (B) after injury, and these effects are completely reversed after acute statin withdrawal. Using $\mathrm{FeCl}_{3}$, carotid injury was induced in 20 -week-old apoE-/- mice after 2 weeks of treatment with vehicle (control group; $\mathrm{n}=26$ ), I mg/kg body weight (BW) rosuvastatin i.p. (low-dose group; $\mathrm{n}=27$ ), or $10 \mathrm{mg} / \mathrm{kg} \mathrm{BW}$ rosuvastatin (high-dose group; $\mathrm{n}=24$ ). Mice in the withdrawal group $(n=12)$ were treated with high-dose rosuvastatin for 14 days. At that time, the treatment was withdrawn and arterial injury was induced 4 days later. A, The horizontal lines through each grouping correspond to the medians of the displayed values. The $p$ values shown refer to comparison with the control group. B: Bars indicate the proportion of patent vessels in each group at the end of the 25-minute flow-monitoring period after injury. 
(10/26 injured vessels) in control mice, as opposed to $59 \%$ $(16 / 27)$ in the low-dose $(P=0.22)$ and $67 \%(16 / 24)$ in the highdose group $(P=0.08$; Fig. 1B). Thus, rosuvastatin treatment appeared to attenuate the thrombotic response of mouse vessels to endothelial injury by delaying and/or preventing thrombus formation, and by enhancing thrombus resolution and arterial recanalisation.

In further experiments, carotid injury was induced in mice 4 days after withdrawal of high-dose rosuvastatin treatment. In these studies, the percentage of non-occluded vessels was reduced from $38 \%$ in the high-dose group to $17 \%$ in the withdrawal group ( $P=0.27)$. Furthermore, the times to thrombosis after statin withdrawal (15.2 \pm 4.8 minutes; median, 13.4$)$ were no longer significantly different from those in vehicle-treated mice (Fig. 1A). Finally, only 2 of 12 mouse carotid vessels (17\%) in the withdrawal group were patent 30 minutes after injury, a significantly lower proportion compared to rosuvastatin-treated mice ( $P=0.018$ vs. the low-dose and 0.012 vs. the high-dose group), and slightly lower compared to the control group (Fig. 1B). These results suggested that acute withdrawal of high-dose rosuvastatin before induction of endothelial injury completely reversed the antithrombotic effects of the drug.

\section{Rosuvastatin modulates platelet aggregation in vitro}

We examined whether the effects of rosuvastatin on arterial thrombosis were mediated by its effects on platelet function. Platelet-rich plasma was isolated from apoE-/- mice that had been treated with rosuvastatin or with vehicle alone for 2 weeks. Maximum platelet aggregation (i.e., the maximum change in light transmission expressed as percentage of PPP light transmission) in response to high concentrations of ADP (10 mM) was significantly decreased in mice treated with low-dose $(24.3 \%)$ and particularly with high-dose rosuvastatin (7.8\%) compared to control mice $(38.9 \% ; P<0.001$ for both comparisons; not shown).

\section{Rosuvastatin treatment reduces neointimal growth after injury}

After injury with ferric chloride, apoE-/- mice continued to receive rosuvastatin or vehicle by intraperitoneal injection once daily for 3 weeks. At the end of that period, the effects of rosuvastatin treatment on neointimal growth were quantified both haemodynamically and histochemically. Doppler flow studies of anaesthetised mice prior to sacrifice and tissue harvest showed that all $(100 \%)$ injured carotid vessels in the 3 treatment groups were
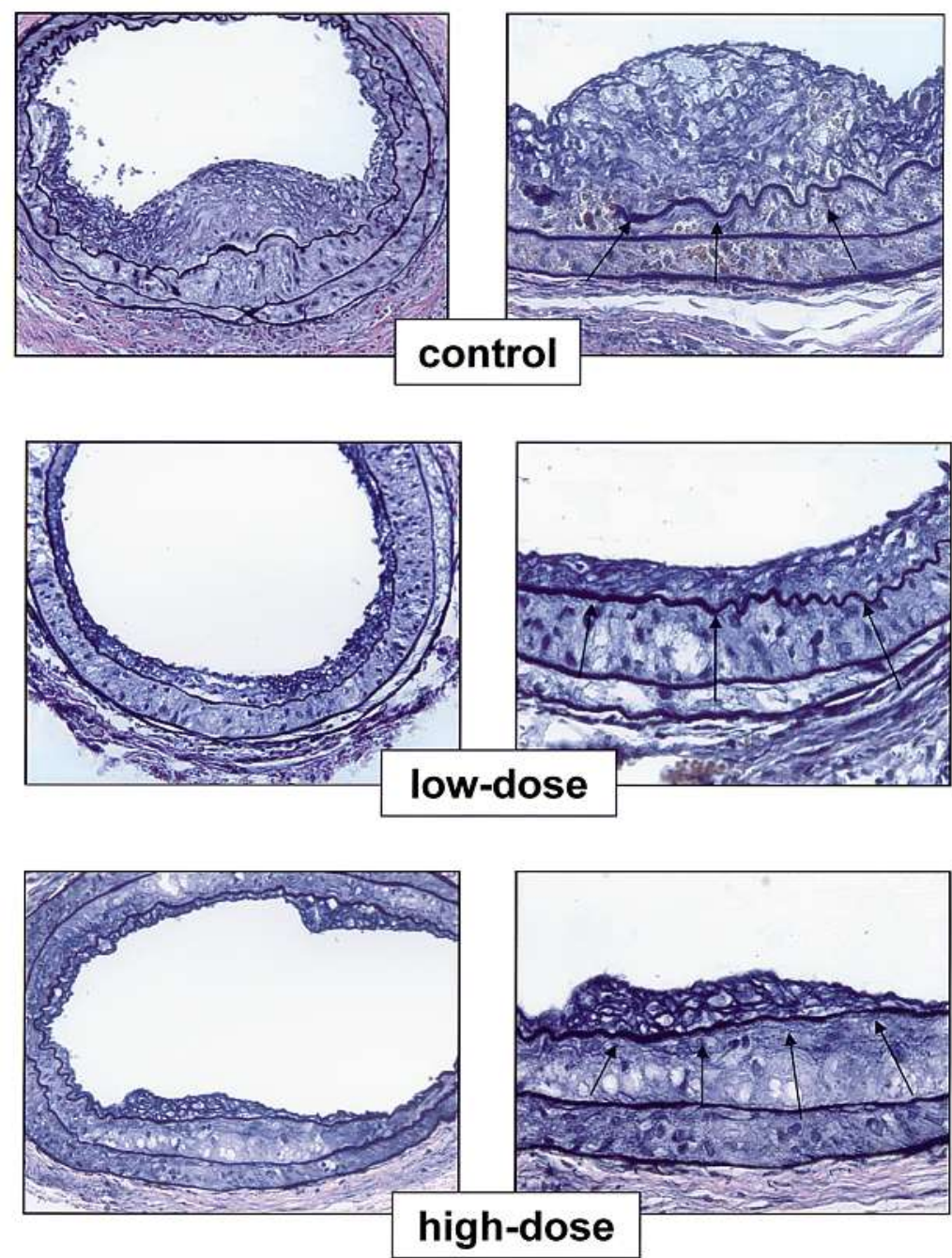

Figure 2: Neointimal growth after arterial injury in apoE-/- mice is reduced by rosuvastatin treatment in a dose-dependent manner. Displayed are representative sections of carotid vessels harvested 3 weeks after injury and stained with Verhoeff's elastic stain. Arrows denote the internal elastic lamina. Magnification, x400 (left panels); and xI000 (right panels). 
patent (not shown), indicating the absence of residual occluding thrombus. However, Verhoeff's elastic stain of paraffin-embedded arterial sections revealed that daily treatment with rosuvastatin at either the low $(1 \mathrm{mg} / \mathrm{kg})$ or the high $(10 \mathrm{mg} / \mathrm{kg})$ dosage reduced the area of the neointima but not that of the media in apoE-/- mice (Fig. 2). Quantitative morphometric analysis confirmed these findings by demonstrating a significant reduction of the intima-to-media ratio from $0.42 \pm 0.03$ in the control group to $0.30 \pm 0.03$ in the low-dose and $0.30 \pm 0.05$ in the high-dose rosuvastatin group (Fig. 3A). In addition, the severity of luminal stenosis resulting from neointimal growth was significantly decreased in both the low-dose $(21.3 \pm 1.9 \%)$ and the high-dose rosuvastastin group $(22.3 \pm 2.9 \%)$ compared to the vehicle-treated mice (32.3 $2.4 \%$; Fig. 3B).

\section{Effects on the composition of vascular lesions}

Quantitative immunohistochemical analysis of the neointima and media demonstrated that rosuvastatin treatment at the time of injury and throughout the 3-week vascular wound healing period was associated with reduced fibrin(ogen) deposition in the vessel wall. This effect was particularly pronounced in the high-dose group, but it was already significant in mice receiving $1 \mathrm{mg} / \mathrm{kg}$ rosuvastatin daily (Fig. 4, A-C; quantitative analysis in D). Interestingly, the reduction in intramural fibrinogen was not accompanied by changes in the protein expression of plasminogen activator inhibitor-1 or tissue factor (not shown). Further immunohistochemical studies revealed a reduction in Mac-3-immunopositive area from $70.8 \pm 5.5 \%$ in the control group to $65.5 \pm 7.9 \%$ in the low-dose and $42.4 \pm 9.8 \%$ in the highdose group. This finding reflected a reduction in the number of Mac-3-positive macrophages in the vessel wall (not shown). Importantly, since most of these cells were identified as foam cells which stained positive for oxLDL, rosuvastatin was also dosedependently associated with a reduction in the oxLDL-positive area in the vessel wall (Fig. 5, A-C; analysis in D) (21). Finally, statin treatment was accompanied by an increase both in the smooth muscle cell content (from $5.2 \pm 0.7 \%$ in the control group to $8.2 \pm 2.1 \%$ in the low-dose and $11.8 \pm 2.4 \%$ in the high-dose group; $P=0.008$ and $P<0.001$, respectively) and in the collagen content (from $4.8 \pm 2.4 \%$ in the control group to $6.2 \pm 0.9 \%$ in the low-dose and $12.9 \pm 1.7 \%$ in the high-dose group; $P=0.26$ and $P<0.001$, respectively) of arterial lesion ns developing 3 weeks after injury (not shown).

\section{Discussion}

Studies on human atherosclerosis suggest that treatment with statins not only reduces the size of lesions and the thickness of the arterial wall, but that it may also contribute to plaque stabilisation by decreasing lipid content, inflammation and matrix proteolysis while increasing collagen content $(25,26)$. These observations, which are in agreement with the results obtained in a rabbit model of hyperlipidaemia and atherosclerosis (27), have largely been attributed to the established lipid-lowering effects of statins in humans and most animal models. In fact, dietary lipid lowering results in histological changes, similar to those described after statin treatment $(23,27)$, and the physiological importance of the so-called pleiotropic effects of statins on the

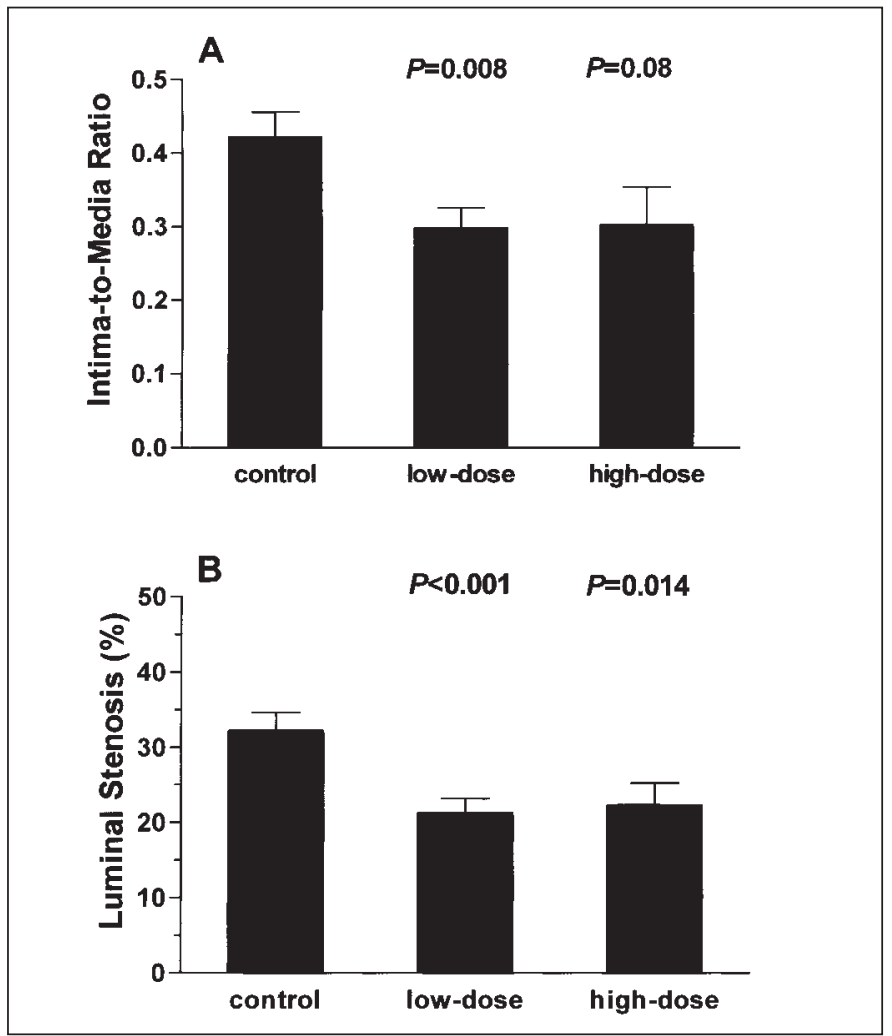

Figure 3: Effects of low-dose and high-dose statin treatment on the intima-to-media ratio and the degree of luminal stenosis in apoE-/- mice 3 weeks after arterial injury: quantitative morphometric analysis. Five sections equally spaced throughout the injured segment (at 200- $\mu \mathrm{m}$ intervals) were evaluated and the results were averaged for each animal. Mean values \pm S.E.M. were calculated from 18 control mice, 21 mice in the low-dose and 19 mice in the high-dose group. The $P$ values shown above the bars refer to the comparison with the control group.

vessel wall remains speculative to date (5). Convincing evidence for the presence of direct anti-atherosclerotic (i.e. anti-inflammatory and plaque-stabilising) effects of statins independent of systemic lipid lowering could have enormous clinical implications, since it might extend the use of these drugs to primary prevention of cardiovascular disease in a large population of high- and intermediate-risk normolipidaemic individuals (28).

In vivo models for systematically analysing the effects of statin treatment on vascular biology have thus far focused either on stroke protection following ligation of the middle cerebral artery $(29,30)$, or on neointimal growth after mechanical (wire- or balloon-induced) injury to the carotid artery $(31,32)$. In the present study, we used the ferric chloride model of carotid artery injury which reproducibly induces the formation of platelet-rich occluding thrombi with subsequent fibrin deposition, thrombus organisation and neointimal formation $(15,21,22)$. As this sequence of events partly reproduces critical pathophysiological steps in atherosclerosis and atherothrombosis (16), we anticipated that the ferric chloride model might allow us to study the effects of statin treatment on various cell types including platelets, endothelial cells, smooth muscle cells, and macrophages. In agreement with previous observations in apoE-/- and LDL-re- 

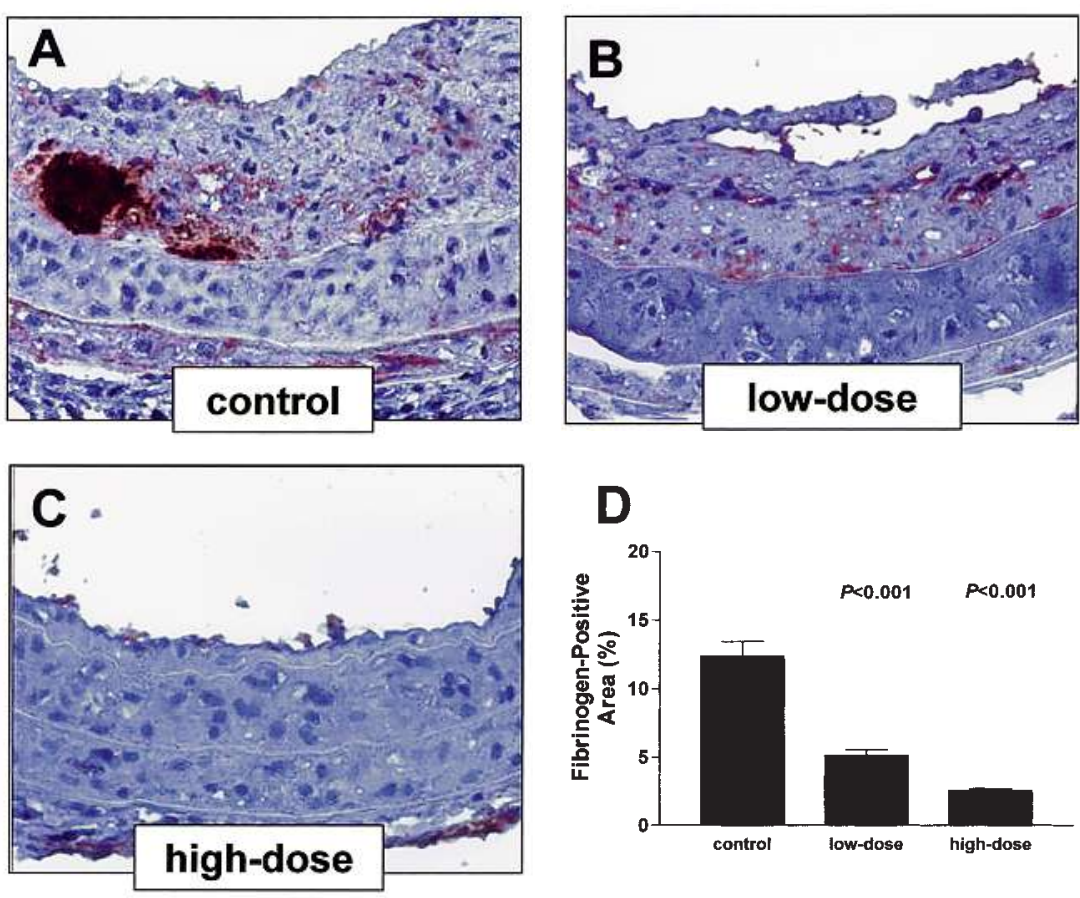

Figure 4: Rosuvastatin dose-dependently reduces fibrin(ogen) deposition in injured carotid vessels. A-C, Representative sections analysed immunohistochemically for the presence of fibrin(ogen) (brown stain; magnification, x 1000). D: Quantitative analysis of immunoreactive (fibrinogen-positive) area as percentage of total vessel wall area within the external elastic lamina. Bars represent means \pm S.E.M. of measurements performed in 6 mice per group.. ceptor-knockout mice $(9,10,31)$ and in contrast to recently reported studies in apoE3*-Leiden transgenic mice (33), we found no significant effects of rosuvastatin treatment on body weight or the lipid profile of mice. Despite the persistence of hypercholesterolaemia, a known potent stimulus of platelet aggregation and thrombosis (18), our in vivo experiments revealed that rosuvastatin treatment significantly attenuated the thrombotic response of apoE-/- mice to arterial injury in a dose-dependent manner. These results were associated with a reduction in the in vitro aggregation of platelet-rich plasma from rosuvastatintreated mice in response to ADP. Our findings thus extend previous observations in normocholesterolaemic mice (34) and add further support to the hypothesis that statins possess important antithrombotic properties which may extend beyond antagonisation of the prothrombotic effects of hypercholesterolaemia $(35,36)$. In this regard, it is likely that statins may directly inhibit
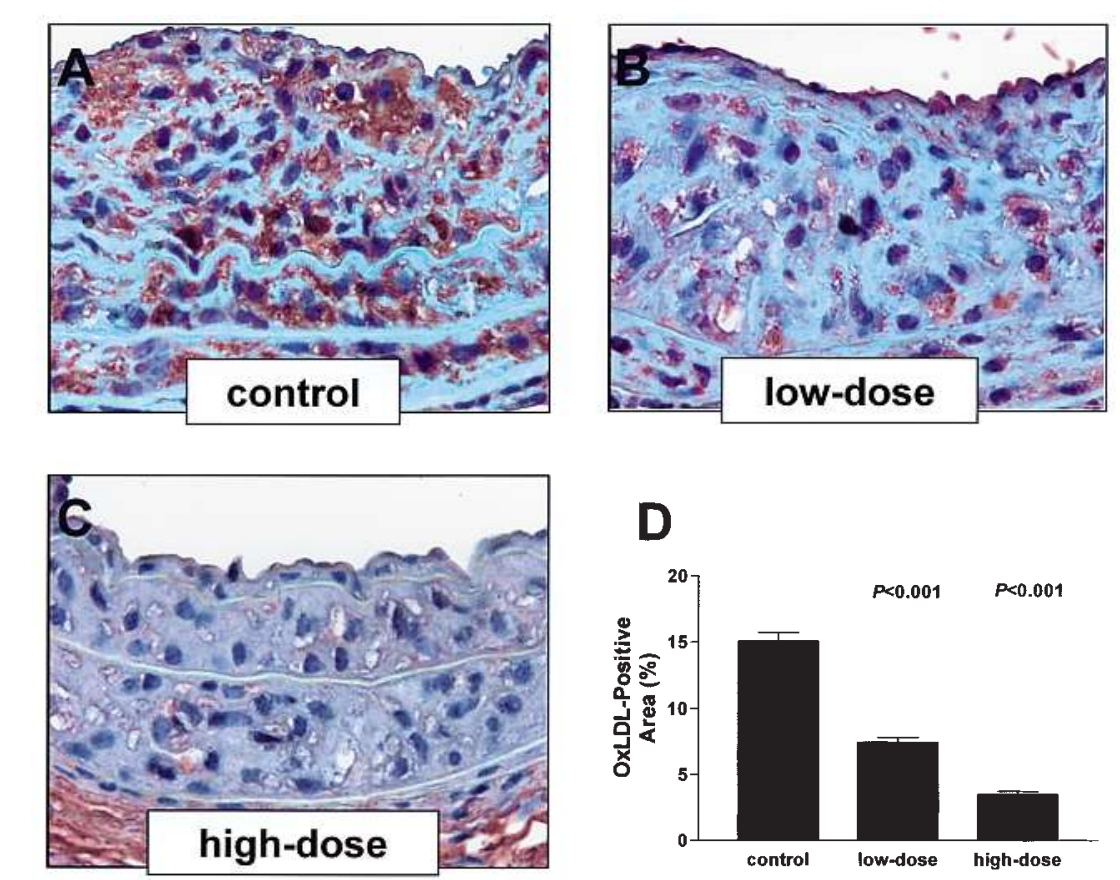

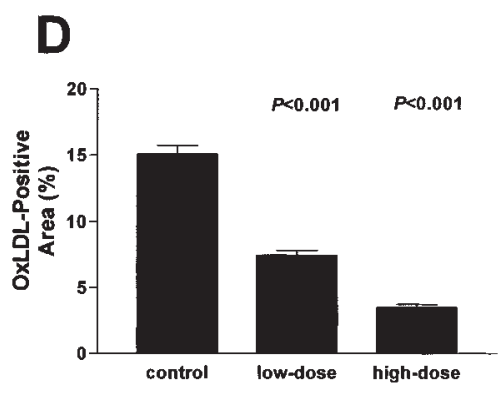

Figure 5: The number of lipid-laden macrophages in the neointima and media of injured vessels is significantly reduced by low and particularly by high doses of rosuvastatin. A-C, Representative sections examined immunohistochemically for oxLDL (pink cells; magnification, xl000). D: Quantitative analysis of immunoreactive (oxLDL-positive) area as percentage of total vessel wall area. Bars represent means + S.E.M. of measurements performed in 6 mice per group. 
platelet activation by upregulating endothelial nitric oxide synthase in these cells (34). Importantly, the protective effects of statins on platelet function may be reversed 2-4 days after acute statin withdrawal (37), and we could in fact show in the present study that abrupt cessation of rosuvastatin treatment resulted in complete restoration of the thrombotic response to injury and the fast formation of stable, lysis-resistant arterial thrombi. At present, it remains to be determined whether statin withdrawal in patients with acute coronary syndromes may significantly increase the risk for cardiovascular morbidity and mortality (38).

Following induction of arterial injury, low-dose or high-dose rosuvastatin (or vehicle) treatment was continued in our study throughout the 3 -week wound healing period (21). In agreement with a recent report in LDL-receptor-knockout mice (31), statin treatment reduced the size of vascular lesions (i.e. the intimamedia ratio and the severity of luminal stenosis) developing after injury. These effects, which were already significant at low (1 $\mathrm{mg} / \mathrm{kg}$ ) dosages of rosuvastatin, may be associated with accelerated reendothelialisation following mobilisation and incorporation of bone marrow-derived endothelial progenitor cells (EPCs) $(32,39)$. Of note, an increase in the number of circulating EPCs under statin treatment has also been observed in patients with coronary disease (40), emphasizing that the potential role of EPCs in the pathophysiology of the vascular response to injury deserves further investigation. Alternatively, or in addition to this mechanism, our results may be due to the significant reduction of thrombus size and subsequent fibrin deposition in the vessel wall of rosuvastatin-treated mice. In fact, we and others have shown that certain injury models, including the ferric chloride model used in the present study, are characterised by a marked thrombotic reaction and fibrin deposition in the arterial wall (21, $41,42)$. In these models and in human atherosclerosis $(15,16)$, organising thrombus may provide an important provisional matrix for cell migration and neointimal growth.

A further finding of our study was that daily rosuvastatin treatment during the vascular remodelling process not only affected lesion size but also reduced the accumulation of oxLDLladen macrophages (foam cells) and increased the $\alpha$-actin (smooth muscle cell) and collagen content of the plaques in a dose-dependent manner. These results add to the evidence supporting the anti-inflammatory and plaque-stabilising effects of statins in vivo $(10,26,27,33)$ and also suggest that, at least in the mouse, these effects are independent of systemic lipid lowering. Of note, previous studies demonstrated that statins attenuated the proliferation of cultured smooth cells by interfering with $\mathrm{G}$ protein-mediated cell cycle regulation (43) and raised fears that a reduction in the smooth muscle cell population might contribute to plaque destabilisation and rupture. However, this concern is probably not justified in the clinical setting (5), since the concen- trations of statins required to inhibit smooth muscle cell proliferation in vitro are rarely, if ever, achieved in vivo. In fact, our results support the thesis that the beneficial anti-inflammatory effects of statins are those that predominate and contribute to increased plaque stability following vascular injury in vivo.

We also found that the change in composition of vascular lesions in rosuvastatin-treated apoE-/- mice was not accompanied by a decrease in the local expression of plasminogen activator inhibitor-1 or tissue factor. This finding was unexpected in light of previous in vitro data $(44,45)$. Moreover, it was recently reported that administration of simvastatin for up to 24 weeks reduced tissue factor expression in atherosclerotic plaques of apoE-/- mice (46). The explanation for the apparent discrepancy between this latter study and our own results may be the differences in the type and dosage of statin used, the duration of treatment, and, particularly, the type of vascular lesions studied (i.e., spontaneously developing atherosclerotic plaques vs. neointimal lesions after ferric chloride-induced endothelial injury). Clearly, caution is warranted when extra-polating in vitro effects of statins on cultured endothelial or smooth muscle cells to pathophysiological processes in vivo and particularly to human disease. It also needs to be mentioned that experimental mouse models of atherosclerosis and/or arterial injury, including the ferric chloride model used in the present study, cannot exactly reproduce the pathophysiology of human atherosclerosis, atherothrombosis, or restenosis. Notwithstanding this limitation, lesions developing in mice after injury with ferric chloride exhibit several histological characteristics of human atherosclerotic plaques $(15,19)$, and their systematic study helps dissect basic pathomechanisms of the vascular wound healing (remodelling) process in the presence of cardiovascular risk factors such as hyperlipidaemia (18).

In conclusion, our studies in apoE-/- mice reveal that rosuvastatin attenuates platelet aggregation and the thrombotic response to endothelial injury, reduces neointimal growth and the size of vascular lesions, and favourably alters the composition of plaques by reducing fibrin deposition and foam cell accumulation while increasing smooth muscle cell and collagen content. Since these effects were not associated with a concomitant improvement in the lipid profile of mice, our results strengthen the existing experimental evidence favouring the pleiotropic effects of statins on thrombocytes and the vessel wall, and they thus support the need for clinical trials to test the benefits of statins in the primary prevention of cardiovascular events in appropriately selected normolipidaemic individuals.

\section{Acknowledgements}

We appreciate the technical assistance of Colin Goeschen, Marianne Pynn and Sandra Skoro.

\section{References}

1. Maron DJ, Fazio S, Linton MF. Current perspectives on statins. Circulation 2000; 101: 207-13.

2. Executive Summary of The Third Report of The National Cholesterol Education Program (NCEP) Expert Panel on Detection, Evaluation, And Treatment of High Blood Cholesterol In Adults (Adult Treatment Panel III). JAMA 2001; 285: 2486-97.
3. Palinski W. New evidence for beneficial effects of statins unrelated to lipid lowering. Arterioscler Thromb Vasc Biol 2001; 21: 3-5.

4. Kwak B, Mulhaupt F, Myit S, et al. Statins as a newly recognized type of immunomodulator. Nat Med 2000; 6: 1399-402.
5. Libby P. Current concepts of the pathogenesis of the acute coronary syndromes. Circulation 2001; 104 : 365-72.

6. MRC/BHF Heart Protection Study of cholesterol lowering with simvastatin in 20,536 high-risk individuals: a randomised placebo-controlled trial. Lancet 2002; 360: 7-22. 
7. Schwartz GG, Olsson AG, Ezekowitz MD, et al. Effects of atorvastatin on early recurrent ischemic events in acute coronary syndromes: the MIRACL study: a randomized controlled trial. JAMA 2001; 285: 1711-8.

8. Scalia R, Gooszen ME, Jones SP, et al. Simvastatin exerts both anti-inflammatory and cardioprotective effects in apolipoprotein E-deficient mice. Circulation 2001; 103: 2598-603.

9. Sparrow CP, Burton CA, Hernandez M, et al. Simvastatin has anti-inflammatory and anti-atherosclerotic activities independent of plasma cholesterol lowering. Arterioscler Thromb Vasc Biol 2001; 21: 115-21.

10. Bea F, Blessing E, Bennett B, et al. Simvastatin promotes atherosclerotic plaque stability in apoE-deficient mice independently of lipid lowering. Arterioscler Thromb Vasc Biol 2002; 22: 1832-7.

11. Wang YX, Martin-McNulty B, Huw LY, et al Anti-atherosclerotic effect of simvastatin depends on the presence of apolipoprotein E. Atherosclerosis 2002; 162: 23-31.

12. Luttun A, Lupu F, Storkebaum E, et al. Lack of plasminogen activator inhibitor-1 promotes growth and abnormal matrix remodeling of advanced atherosclerotic plaques in apolipo-protein E-deficient mice. Arterioscler Thromb Vasc Biol 2002; 22: 499-505.

13. Eitzman DT, Westrick RJ, Xu Z, et al. Plasminogen activator inhibitor-1 deficiency pro-tects against atherosclerosis progression in the mouse carotid artery. Blood 2000; 96: 4212-5.

14. Sjoland H, Eitzman DT, Gordon D, et al. Atherosclerosis progression in LDL receptor-deficient and apolipoprotein E-deficient mice is independent of genetic alterations in plasminogen activator inhibitor- 1 . Arterioscler Thromb Vasc Biol 2000; 20: 846-52.

15. Schäfer K, Konstantinides S, Riedel C, et al. Different mechanisms of increased luminal stenosis after arterial injury in mice deficient for urokinase- or tissue-type plasminogen activator. Circulation 2002; 106: 1847-52.

16. Burke AP, Kolodgie FD, Farb A, et al. Healed plaque ruptures and sudden coronary death : evidence that subclinical rupture has a role in plaque progression. Circulation 2001; 103: 934-40.

17. Libby P, Simon DI. Inflammation and thrombosis : the clot thickens. Circulation 2001; 103: 1718-20.

18. Schäfer K, Müller K, Hecke A, et al. Enhanced thrombosis in atherosclerosis-prone mice is associated with increased arterial expression of plasminogen activator inhibitor-1. Arterioscler Thromb Vasc Biol 2003; 23: 2097-103.

19. Zhu Y, Farrehi PM, Fay WP. Plasminogen activator inhibitor type 1 enhances neointima formation after oxidative vascular injury in atherosclerosis-prone mice. Circulation 2001; 103: 3105-10.

20. Paoletti R, Fahmy M, Mahla G, et al. Rosuvastatin demonstrates greater reduction of low-density lipoprotein cholesterol compared with pravastatin and simvastatin in hypercholesterolaemic patients: a randomized, double-blind study. J Cardiovasc Risk 2001; 8: 383-90. 21. Konstantinides S, Schäfer K, Thinnes T, et al. Plasminogen activator inhibitor- 1 and its cofactor vitronec- tin stabilize arterial thrombi after vascular injury in mice. Circulation 2001; 103: 576-83.

22. Pynn M, Schäfer K, Konstantinides S, et al. Exercise training reduces neointimal growth and stabilizes vascular lesions developing after injury in apolipoprotein E-deficient mice. Circulation 2004; 109: 386-92. 23. Aikawa M, Rabkin E, Okada Y, et al. Lipid lowering by diet reduces matrix metalloproteinase activity and increases collagen content of rabbit atheroma: a potential mechanism of lesion stabilization. Circulation 1998; 97: 2433-44.

24. Konstantinides S, Schäfer K, Koschnick S, et al. Leptin-dependent platelet aggregation and arterial thrombosis suggests a mechanism for atherothrombotic disease in obesity. J Clin Invest 2001; 108: 1533-40.

25. Corti R, Fayad ZA, Fuster V, et al. Effects of lipidlowering by simvastatin on human atherosclerotic lesions: a longitudinal study by high-resolution, noninvasive magnetic resonance imaging. Circulation 2001; 104: 249-52.

26. Crisby M, Nordin-Fredriksson G, Shah PK, et al. Pravastatin treatment increases collagen content and decreases lipid content, inflammation, metalloproteinases, and cell death in human carotid plaques : implications for plaque stabilization. Circulation 2001; 103: 926-33.

27. Aikawa M, Rabkin E, Sugiyama S, et al. An HMG$\mathrm{CoA}$ reductase inhibitor, cerivastatin, suppresses growth of macrophages expressing matrix metalloproteinases and tissue factor in vivo and in vitro. Circulation 2001; 103: 276-83.

28. Ridker PM. Rosuvastatin in the primary prevention of cardiovascular disease among patients with low levels of low-density lipoprotein cholesterol and elevated high-sensitivity C-reactive protein: rationale and design of the JUPITER trial. Circulation 2003; 108: $2292-7$.

29. Laufs U, Gertz K, Dirnagl U, et al. Rosuvastatin, a new HMG-CoA reductase inhibitor, upregulates endothelial nitric oxide synthase and protects from ischemic stroke in mice. Brain Res 2002; 942: 23-30. 30. Laufs U, Endres M, Custodis F, et al. Suppression of endothelial nitric oxide production after withdrawal of statin treatment is mediated by negative feedback regulation of rho GTPase gene transcription. Circulation 2000; 102: 3104-10.

31. Chen Z, Fukutomi T, Zago AC, et al. Simvastatin reduces neointimal thickening in low-density lipoprotein receptor-deficient mice after experimental angioplasty without changing plasma lipids. Circulation 2002; 106: 20-3.

32. Werner N, Priller J, Laufs U, et al. Bone marrowderived progenitor cells modulate vascular reendothelialization and neointimal formation: effect of 3-hydroxy-3-methylglutaryl coenzyme a reductase inhibition. Arterioscler Thromb Vasc Biol 2002; 22: 1567-72.

33. Kleemann R, Princen HM, Emeis JJ, et al. Rosuvastatin reduces atherosclerosis development beyond and independent of its plasma cholesterol-lowering effect in APOE*3-Leiden transgenic mice: evidence for antiinflammatory effects of rosuvastatin. Circulation 2003; 108: 1368-74.

34. Laufs U, Gertz K, Huang P, et al. Atorvastatin upregulates type III nitric oxide synthase in thrombocytes, decreases platelet activation, and protects from cerebral ischemia in nor-mocholesterolemic mice. Stroke 2000; 31: 2442-9.

35. Cipollone F, Mezzetti A, Porreca E, et al. Association between enhanced soluble CD40L and prothrombotic state in hypercholesterolemia: effects of statin therapy. Circulation 2002; 106: 399-402.

36. Rosenson RS, Tangney CC. Antiathero-thrombotic properties of statins: implications for cardiovascular event reduction [see comments]. JAMA 1998; 279: $1643-50$.

37. Gertz K, Laufs U, Lindauer U, et al. Withdrawal of statin treatment abrogates stroke protection in mice. Stroke 2003; 34: 551-7.

38. Heeschen C, Hamm CW, Laufs U, et al. Withdrawal of statins in patients with acute coronary syndromes. Circulation 2003; 107: e27.

39. Walter DH, Rittig K, Bahlmann FH, et al. Statin therapy accelerates reendothelialization: a novel effect involving mobilization and incorporation of bone marrow-derived endothelial progenitor cells. Circulation 2002; 105: 3017-24.

40. Vasa M, Fichtlscherer S, Adler K, et al. Increase in circulating endothelial progenitor cells by statin therapy in patients with stable coronary artery disease. Circulation 2001; 103: 2885-90.

41. Kawasaki T, Dewerchin M, Lijnen HR, et al Mouse carotid artery ligation induces platelet-leukocyte-dependent luminal fibrin, required for neointima development. Circ Res 2001; 88: 159-66.

42. Ploplis VA, Cornelissen I, Sandoval-Cooper MJ, et al. Remodeling of the vessel wall after copper-induced injury is highly attenuated in mice with a total deficiency of plasminogen activator inhibitor-1. Am J Patho 2001; 158: 107-17.

43. Laufs U, Marra D, Node K, et al. 3-Hydroxy3-methylglutaryl-CoA reductase inhibitors attenuate vascular smooth muscle proliferation by preventing rho GTPase-induced down-regulation of p27(Kip1). J Biol Chem 1999; 274: 21926-31.

44. Eto M, Kozai T, Cosentino F, et al. Statin prevents tissue factor expression in human endothelial cells: role of Rho/Rho-kinase and Akt pathways. Circulation 2002; 105: 1756-9.

45. Bourcier T, Libby P. HMG CoA reductase inhibitors reduce plasminogen activator inhibitor- $1 \mathrm{ex}$ pression by human vascular smooth muscle and endothelial cells. Arterioscler Thromb Vasc Biol 2000; 20: 556-62.

46. Bea F, Blessing E, Shelley MI, et al. Simvastatin inhibits expression of tissue factor in advanced atherosclerotic lesions of apolipoprotein $\mathrm{E}$ deficient mice independently of lipid lowering: potential role of simvastatin-mediated inhibition of Egr-1 expression and activation. Atherosclerosis 2003; 167: 187-94. 